\title{
Bispectral Analysis for Measuring Energy-Orientation Tradeoffs in the Control of Linear Systems
}

\author{
Delsin Menolascino ${ }^{1}$, ShiNung Ching ${ }^{2}$
}

\begin{abstract}
Many characterizations of linear system controllability revolve around the eigenvalue spectrum of the controllability gramian, which is a function of the network dynamics. The gramian spectrum describes the minimum energies associated with inducing movement along orthogonal directions in $\mathbb{R}^{n}$. Here, we derive an enhanced interpretation of the spectral properties of the gramian in nonminimum energy regimes. Indeed, in a non-minimum energetic regime, an 'excess' of energy is available to the system for at least $(n-1)$ orthogonal state transfers. We show that the utility of this excess energy can be quantified in terms of input orientation, or, simply, the angle between two competing inputs. Based on this notion, we derive the gramian bispectrum, which describes the relationship between energy and orientation among pairs of orthogonal state transfers. The bispectrum reflects a fundamental tradeoff between the energetic and orientation costs in the control of a linear system. We show how this bispectral analysis can provide control characterizations that are not apparent from inspection of the gramian spectrum alone.
\end{abstract}

Keywords: network analysis, network control, linear systems, bispectrum

\footnotetext{
${ }^{1}$ The authors are with the Department of Electrical and Systems Engineering, Washington University in Saint Louis, Saint Louis, MO 63130, U.S.A.

${ }^{2}$ Corresponding author: ShiNung Ching is with the Department of Electrical and Systems Engineering \& Division of Biology and Biomedical Sciences, Washington University in Saint Louis, Saint Louis, MO 63130, U.S.A.
}

Preprint submitted to Systems and Control Letters

December 8, 2016

(C) 2017. This manuscript version is made available under the Elsevier user license http://www.elsevier.com/open-access/userlicense/1.0/ 


\section{Introduction}

\subsection{Motivation}

A long-standing topic in control theory, with recent applications in network control [1, 2, 3], involves the development of analyses to quantify the controllability of linear systems [4, 5, 6]. Many of the approaches that have been developed to address this issue involve study of the spectral properties of the controllability gramian, which, for a controllable linear time-invariant system in the typical form

$$
\dot{\mathbf{x}}(t)=\mathbf{A} \mathbf{x}(t)+\mathbf{B u}(t)
$$

is defined as

$$
\mathbf{W}(T)=\int_{0}^{T} \mathrm{e}^{\mathbf{A}(T-\tau)} \mathbf{B}(\tau) \mathbf{B}^{\prime}(\tau) \mathrm{e}^{\mathbf{A}^{\prime}(T-\tau)} \mathrm{d} \tau .
$$

Each eigenvalue of $\mathbf{W}^{-1}$ determines the energy required to induce motion in in the direction of its associated eigenvector. Thus, summary metrics that describe

5 the energetic costs of controlling a linear system can be derived from the gramian spectrum. Such metrics include the trace of the gramian [7, [8, the log of its determinant [7, and the maximum eigenvalue of the inverse gramian [7], 8]. The latter, in particular, is the 'worst-case' minimum energy required to reach the unit hypersphere at a prescribed time $T$.

In this paper we seek to characterize the controllability of a system assuming that we have at least this minimum energy available to meet control objectives. That is, we assume that the entire unit hypersphere is reachable at time $T$. What then, can be said about the system under consideration? For a nonuniform gramian spectrum, there will be a gradation of energetic costs, so that we may steer the system in other directions (than the 'worst-case' direction) with less (or much less) energy expenditure. To what use, then, is the excess energy which we have available? Is there a measure of control flexibility that can be realized under this 'surplus energy' scenario?

To answer these questions we consider, in addition to energy, the relative orientation between two inputs $\mathbf{u}_{1}(t)$ and $\mathbf{u}_{2}(t)$, which transfer the state of the 
system to two different endpoints at time $T$. Assuming these inputs possess average energy $\gamma_{\mathbf{u}_{1}}$ and $\gamma_{\mathbf{u}_{2}}$, then over the time horizon $[0, T]$ the relative orientation, or, mathematically, the average cosine of the angle between $\mathbf{u}_{1}(t)$ and $\mathbf{u}_{2}(t)$, is:

$$
\frac{1}{T \sqrt{\gamma_{\mathbf{u}_{1}} \gamma_{\mathbf{u}_{2}}}} \int_{0}^{T} \mathbf{u}_{1}^{\prime}(\tau) \mathbf{u}_{2}(\tau) \mathrm{d} \tau,
$$

If $\gamma_{\mathbf{u}_{1}}$ and $\gamma_{\mathbf{u}_{2}}$ are minimal for the transfers in question, then (3) is fixed since

${ }_{20} \mathbf{u}_{1}(t)$ and $\mathbf{u}_{2}(t)$ can assume only one form. Given excess energy, inputs achieving the transfers may assume a range of possible relative orientations. We propose that this range constitutes a measure of the aforementioned control flexibility, and we provide an analytical development that makes this notion concrete. Specifically, in this paper we make the following contributions:

1. As a function of available energy, we derive the minimum relative orientation (or, average angle) between two inputs, each inducing a transfer to points along a different eigenvector of $\mathbf{W}^{-1}$. A small relative orientation implies that the inputs are relatively flexible in their orientation range. Indeed, we show that as energy tends to infinity the angular difference tends to zero, so that the inputs may become arbitrarily similar in their geometry. The derivation leverages our previous optimal control results in [9] and [10].

2. We derive a second-order spectrum, termed the gramian bispectrum that exactly quantifies the tradeoff between energy and orientation, in the above sense, for each pair of eigenvalues of the gramian.

3. We demonstrate how the proposed bispectrum may be used to compare the relative utility of energy in linear systems. We provide examples of such comparisons that show how a bispectral analysis may reveal control properties not apparent from the spectrum alone.

40 2. Problem Formulation

\subsection{Geometric Interpretation of the Controllability Gramian}




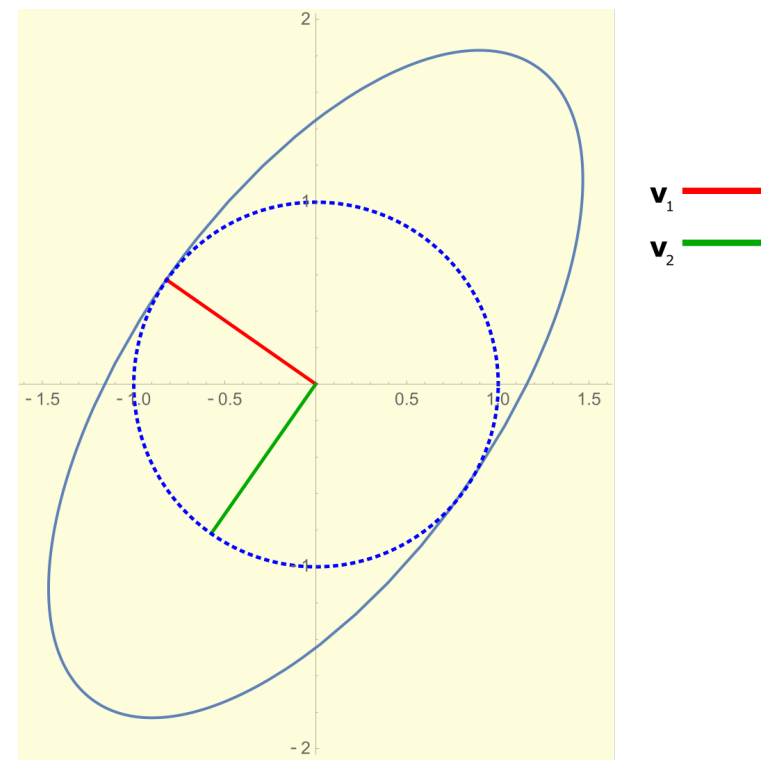

Figure 1: Unit circle with ellipse prescribed by $\mathbf{W}(T)^{-1}$ of a stable two-dimensional system with the two eigenvectors of $\mathbf{W}(T)^{-1}$ Since the ellipse represents the reachable set with fixed energy $\lambda_{1}$, the distance between the ellipse and circle, in the direction of $\mathbf{v}_{2}$, encodes the amount of excess energy available if we desire to steer the system to $\mathbf{v}_{2}$. Note that the figure represents an abstract state space for a 2-d linear system, and thus the axes have no explicit units. 
Our subsequent development will pivot on the spectrum $\Lambda$ of the controllability gramian inverse $\mathbf{W}^{-1}$ (see (2)), defined as the collection of its eigenvalues with ordered labeling, i.e.,

$$
\Lambda=\left\{\lambda_{1}, \ldots, \lambda_{n}\right\}, \lambda_{1} \geq \lambda_{2} \geq \ldots \geq \lambda_{n}
$$

and corresponding eigenvectors $\mathbf{v}_{1}, \ldots, \mathbf{v}_{n}$.

Since the minimum-energy cost of driving the system from the origin at time $t=0$ to final point $\mathbf{x}_{f}$ at time $t=T$ is given by $c\left(\mathbf{x}_{f}\right)=\int_{0}^{T} \mathbf{u}^{\prime}(\tau) \mathbf{u}^{\prime}(\tau) \mathrm{d} \tau=$ $\mathbf{x}_{f}^{\prime} \mathbf{W}^{-1}(T) \mathbf{x}_{f}$, for a fixed energy $c\left(\mathbf{x}_{f}\right)=c^{*}$ the gramian essentially prescribes a reachable ellipsoid in $n$-dimensional space, with vertices located at $\lambda_{1} \mathbf{v}_{1}, \ldots, \lambda_{n} \mathbf{v}_{n}$. This ellipsoid we formally define as

$$
\Xi \equiv\left\{\mathbf{x} \in \mathbb{R}^{n} \mid \mathbf{x}^{\prime} \mathbf{W}^{-1}(T) \mathbf{x}=c^{*}\right\}
$$

Thus, the surface of $\Xi$ encodes the maximal distance to which trajectories can attain at time $T$ under inputs with total energy $c^{*}$ (starting from the origin).

45 A common way to assay the controllability of a linear system is to deduce the minimum energy, $c^{\text {min }}$, so that system is guaranteed access to a unit-radius hypersphere in $\mathbb{R}^{n}$ (i.e., min $c^{*}$ such that $\Xi \supset\left\{\mathbf{x} \in \mathbb{R}^{n} \mid \mathbf{x}^{\prime} \mathbf{x}=1\right\}$ ), which as follows from (5), is simply $\lambda_{1}$ in (4).

However, as posed in the Introduction, in this scenario, most of the hypersphere is reachable with an excess of energy and we would like to quantify the utility of this excess. Carrying forth the geometric interpretation, it is perhaps intuitive to propose a quantification involving the ratio $\lambda_{i} / \lambda_{1}, i=2, \ldots, n$, i.e., the eccentricity of the ellipse made by intersecting $\Xi$ with the $\left(v_{1}, v_{i}\right)$-plane.

As we will show, this quantity not only results in a useful metric for excess energy utilization, but has a precise interpretation in terms of the relative orientation attainable by putative inputs to the system at hand. We first make concrete the notion of orientation range.

\subsection{Input Orientation and Orientation Range}

We begin by defining formally the expression for relative orientation between two inputs, introduced in (3). For our purposes we will assume one of the inputs 
is known a priori.

Definition 1 (Input Orientation). For reference input $\mathbf{u}_{1}(t)$ and 'free' input $\mathbf{u}_{2}(t)$ guiding a system of the form (1) over the time interval $[0, T]$, we define the relative orientation $\mathbf{d}_{\mathbb{J}}\left(\mathbf{u}_{1}(t), \mathbf{u}_{2}(t), t \in[0, T]\right)$-for ease of notation simplified to $\mathbf{d}_{\mathbb{J}}\left(\mathbf{u}_{1}, \mathbf{u}_{2}\right)$-as follows:

$$
\mathbf{d}_{\mathbb{J}}\left(\mathbf{u}_{1}, \mathbf{u}_{2}\right)=\frac{1}{T \sqrt{\gamma_{\mathbf{u}_{1}} \gamma_{\mathbf{u}_{2}}}} \int_{0}^{T} \mathbf{u}_{1}^{\prime}(\tau) \mathbf{u}_{2}(\tau) \mathrm{d} \tau,
$$

where

$$
\gamma_{\mathbf{u}_{1}}=\frac{1}{T} \int_{0}^{T} \mathbf{u}_{1}^{\prime}(\tau) \mathbf{u}_{1}(\tau) \mathrm{d} \tau, \quad \gamma_{\mathbf{u}_{2}}=\frac{1}{T} \int_{0}^{T} \mathbf{u}_{2}^{\prime}(\tau) \mathbf{u}_{2}(\tau) \mathrm{d} \tau
$$

are the average energies (up to time $T$ ) of $\mathbf{u}_{1}(t)$ and $\mathbf{u}_{2}(t)$, respectively.

We again note that, although we refer to $\mathbf{d}_{\mathbb{J}}\left(\mathbf{u}_{1}, \mathbf{u}_{2}\right)$ as the relative orientation between inputs $\mathbf{u}_{1}$ and $\mathbf{u}_{2}$, in a strict mathematical sense it quantifies the average cosine of the angle between the two inputs.

Under our assumption, the reference is known (thus $\gamma_{\mathbf{u}_{1}}$ is known), and we seek, given some chosen available energy $\gamma_{\mathbf{u}_{2}}$, to quantify our freedom in allowing $\mathbf{u}_{2}(t)$ to assume different geometries while still accomplishing a desired state transfer. We now propose a notion of orientation range based on the maximum similarity between two inputs of fixed energy:

Definition 2 (Maximum Similarity). For inputs of energy $\gamma_{\mathbf{u}_{1}}, \gamma_{\mathbf{u}_{2}}$, the maximum similarity is defined as

$$
\begin{array}{ll}
\underset{\mathbf{u}_{2}}{\operatorname{Max}} & \mathbf{d}_{\mathbb{J}}\left(\mathbf{u}_{1}, \mathbf{u}_{2}\right) \\
\text { Subject To } & \mathbf{x}_{1_{f}}=\int_{0}^{T} e^{\mathbf{A}(T-\tau)} \mathbf{B}(\tau) \mathbf{u}_{1}(\tau) d \tau \\
& \mathbf{x}_{2_{f}}=\int_{0}^{T} e^{\mathbf{A}(T-\tau)} \mathbf{B}(\tau) \mathbf{u}_{2}(\tau) d \tau \\
& \frac{1}{T} \int_{0}^{T} \mathbf{u}_{1}^{\prime}(\tau) \mathbf{u}_{1}(\tau) \mathrm{d} \tau=\gamma_{\mathbf{u}_{1}} \\
& \frac{1}{T} \int_{0}^{T} \mathbf{u}_{2}^{\prime}(\tau) \mathbf{u}_{2}(\tau) \mathrm{d} \tau=\gamma_{\mathbf{u}_{2}}
\end{array}
$$


where we require the system to be steered, over the time interval $[0, T]$, from the points $\mathbf{x}_{1_{0}}$ and $\mathbf{x}_{2_{0}}$ to endpoints $\mathbf{x}_{1_{f}}$ and $\mathbf{x}_{2_{f}}$ by inputs $\mathbf{u}_{1}$ and $\mathbf{u}_{2}$, respectively. Further, $\gamma_{\mathbf{u}_{1}}$ and $\gamma_{\mathbf{u}_{2}}$ are energy constraints on the inputs.

Lemma 1. The optimization problem (8) has a provably unique solution $\mathbf{u}_{2}^{*}$, and the minimum relative orientation is given by the following:

$$
\begin{aligned}
& \mathbf{d}_{\mathbb{J}}\left(\mathbf{u}_{1}, \mathbf{u}_{2}^{*}\right)=\frac{1}{T \sqrt{\gamma_{\mathbf{u}_{1}} \gamma_{\mathbf{u}_{2}^{*}}^{*}}} * \\
& {\left[\left(\mathbf{x}_{1_{f}}-\mathrm{e}^{\mathbf{A} T} \mathbf{x}_{1_{0}}\right)^{\prime} \mathbf{W}^{-1}\left(\mathbf{x}_{2_{f}}-\mathrm{e}^{\mathbf{A} T} \mathbf{x}_{2_{0}}\right)\right.} \\
& \left.+\sqrt{\left(\gamma_{\mathbf{u}_{1}} T-\mathbb{E}_{\min _{1}}\right)\left(\gamma_{\mathbf{u}_{2}^{*}} T-\mathbb{E}_{\min _{2}}\right)}\right]
\end{aligned}
$$

where to simplify notation we employ $\mathbf{W}$ to represent $\mathbf{W}(T)$ (see (2)), and where $\mathbb{E}_{\min _{1}}$ and $\mathbb{E}_{\min _{2}}$ are the minimum energies required for state transfers from the origin to $\mathbf{x}_{1_{f}}$ and $\mathbf{x}_{2_{f}}$, respectively.

Proof 1. The existence and uniqueness of $\mathbf{u}_{2}^{*}$ is proved, by deriving optimality conditions based on the Euler-Lagrange equations, in [9], and a derivation of (9) is given in [10].

so Remark 1. A critical conceptual point is that relative orientation is minimized when $\mathbf{d}_{\mathbb{J}}\left(\mathbf{u}_{1}, \mathbf{u}_{2}^{*}\right)=1$. The relative orientation decreases as $\mathbf{d}_{\mathbb{J}}$ increases, since a larger value means that inputs are more similar. This is apparent from (6), applying the law of cosines.

We note that $\mathbf{d}_{\mathbb{J}}\left(\mathbf{u}_{1}, \mathbf{u}_{2}^{*}\right)=\mathbf{d}_{\mathbb{J}}\left(\mathbf{u}_{2}, \mathbf{u}_{1}^{*}\right)$; that is, in considering the general form for relative orientation given in (6), we may choose either $\mathbf{u}_{1}$ or $\mathbf{u}_{2}$ to be the reference input, and the resulting minimum relative orientation (given by (9)) will be equivalent. This is because if we maximize (8) over $\mathbf{u}_{1}$ with respect to $\mathbf{u}_{2}$, the optimization constraints (i.e. those prescribing terminal states $\mathbf{x}_{1}$ and $\mathbf{x}_{2}$ and input energies $\gamma_{\mathbf{u}_{1}}$ and $\gamma_{\mathbf{u}_{2}}$ ) are identical to those in (8), and the order of $\mathbf{u}_{1}$ and $\mathbf{u}_{2}$ does not affect the value of (6). Indeed, aside from $\mathbf{W}, \mathbb{E}_{\min _{1}}$, and $\mathbb{E}_{\min _{2}}$, which are independent of $\mathbf{u}_{1}$ and $\mathbf{u}_{2},(9)$ depends only upon these 
constraints and is invariant to their indexing (that is, we may 'swap' 1's for 2's in (9) without changing the value). Thus we may, without ambiguity, speak of the minimum relative orientation between inputs required for two state transfers, without deciding which input is the reference.

Thus, the solution (see [9] for a derivation and expression for $\mathbf{u}_{2}^{*}$ ) to 9 ) provides a direct characterization of the maximum similarity that two inputs, to two different points in state space, may attain as a function of energy. This solution, thus, provides us with a concrete notion of orientation range. We will now operationalize this notion by sampling, in a systemic way, the space of possible pairs of terminal states $\left(\mathbf{x}_{1}\right.$ and $\left.\mathbf{x}_{2}\right)$ and possible input energies $\gamma_{\mathbf{u}_{1}}$ and $\gamma_{\mathbf{u}_{2}}$. As may be surmised, the sampling proceeds along the spectrum of $\mathbf{W}^{-1}$.

\section{Results}

The expression (9) is notable because it indicates that the minimum orientation difference associated with a pair of state transfers (each starting at the origin) depends only on the energy prescribed to those transfers. That is, while $\mathbf{u}_{1}$ may take many forms (assuming $\gamma_{\mathbf{u}_{1}} T>\mathbb{E}_{\min _{1}}$ ) its minimum difference with respect to any putative $\mathbf{u}_{2}$ with $\gamma_{\mathbf{u}_{2}} T>\mathbb{E}_{\min _{2}}$ is always the same.

\subsection{The Gramian Bispectrum}

Generally, we seek to compare a set of $M n$-dimensional systems (or networks), denoted $S_{k}, k \in\{1 \ldots M\}$. For each system $S_{k}$, we obtain the spectrum of the inverse gramian at some fixed time $T$ (denoted, as above, by $\mathbf{W}^{-1}$ ) to ascertain their energy characteristics. For the bispectral analysis we create, for each $S_{k}$, a matrix $\mathbf{B i s}_{k}$ showing the minimum relative orientation required for pairs of inputs guiding the system from the origin to endpoints on the $n$ dimensional unit hypersphere. These endpoints are simply the (orthonormal) eigenvectors of $\mathbf{W}^{-1}$, ranked in terms of their corresponding eigenvalues. Since each transfer begins at the origin, we can simplify (9) (we also generalize indices) to obtain 


$$
\begin{aligned}
\mathbf{d}_{\mathbb{J}}\left(\mathbf{u}_{i}, \mathbf{u}_{j}\right)=\frac{1}{T \sqrt{\gamma_{\mathbf{u}_{i}} \gamma_{\mathbf{u}_{j}}}} * & \\
& \left(\mathbf{x}_{\mathbf{u}_{i}}^{\prime} \mathbf{W}^{-1} \mathbf{x}_{\mathbf{u}_{j}}+\sqrt{\left(\gamma_{\mathbf{u}_{i}} T-\mathbb{E}_{\min _{i}}\right)\left(\gamma_{\mathbf{u}_{j}} T-\mathbb{E}_{\min _{j}}\right)}\right)
\end{aligned}
$$

for target terminal states $\mathbf{x}_{\mathbf{u}_{i}}$ and $\mathbf{x}_{\mathbf{u}_{j}}$.

To fully specify $\mathbf{B i s}{ }_{k}$ we need to also determine the average energy associated with each state transfer. The natural choice is to allow $\gamma_{\mathbf{u}_{i}}=\gamma_{\mathbf{u}_{j}}=\frac{\lambda_{\max }}{T}$. Thus, the total energy supplied is $\lambda_{\max }$, which, as stated in the Introduction, is the smallest amount of energy that enables access to the entire unit hypersphere.

With these specifications we rewrite 10 , for any pair of state transfers in the directions of $\mathbf{v}_{i}$ and $\mathbf{v}_{j}(i, j \in\{1 \ldots n\})$, as follows

$$
\begin{aligned}
& \mathbf{d}_{\mathbb{J}}\left(\mathbf{u}_{i}, \mathbf{u}_{j}\right)= \frac{1}{T \sqrt{\frac{\lambda_{\max }}{T} \frac{\lambda_{\max }}{T}}} * \\
&\left(\mathbf{v}_{i}^{\prime} \mathbf{W}^{-1} \mathbf{v}_{j}+\sqrt{\left(\frac{\lambda_{\max }}{T} T-\mathbb{E}_{\min _{i}}\right)\left(\frac{\lambda_{\max }}{T} T-\mathbb{E}_{\min _{j}}\right)}\right) \\
& \quad=\frac{1}{\lambda_{\max }}\left(\mathbf{v}_{i}^{\prime} \mathbf{W}^{-1} \mathbf{v}_{j}+\sqrt{\left(\lambda_{\max }-\mathbb{E}_{\min _{i}}\right)\left(\lambda_{\max }-\mathbb{E}_{\min _{j}}\right)}\right)
\end{aligned}
$$

Now we consider $\mathbb{E}_{\min _{i}}$ and $\mathbb{E}_{\min _{j}}$. It is well known [1] that the minimum required energy for a state transfer from the origin to some terminal point $\mathbf{x}(T)$ is given by $\mathbf{x}^{\prime}(T) \mathbf{W}^{-1} \mathbf{x}(T)$ (recall that throughout we are using $\mathbf{W}^{-1}$ to represent $\mathbf{W}(T)^{-1}$ ), and since in our case we have $\mathbf{x}(T)=\mathbf{v}_{i}$ (or $\mathbf{v}_{j}$ ), where $\mathbf{v}_{i}$ is a (normal) eigenvector of $\mathbf{W}^{-1}$, the minimum energy becomes

$$
\mathbb{E}_{\min _{i}}=\mathbf{v}_{i}^{\prime} \mathbf{W}^{-1} \mathbf{v}_{i}=\lambda_{i} \mathbf{v}_{i}^{\prime} \mathbf{v}_{i}=\lambda_{i}
$$

Equivalently, $\mathbb{E}_{\min _{j}}=\lambda_{j}$. Also, we note that

$$
\mathbf{v}_{i}^{\prime} \mathbf{W}^{-1} \mathbf{v}_{j}=\mathbf{v}_{i}^{\prime} \lambda_{j} \mathbf{v}_{j}=\lambda_{j} \mathbf{v}_{i}^{\prime} \mathbf{v}_{j}=\left\{\begin{array}{l}
0 \text { when } i \neq j \\
\lambda_{j} \text { when } i=j
\end{array}\right.
$$



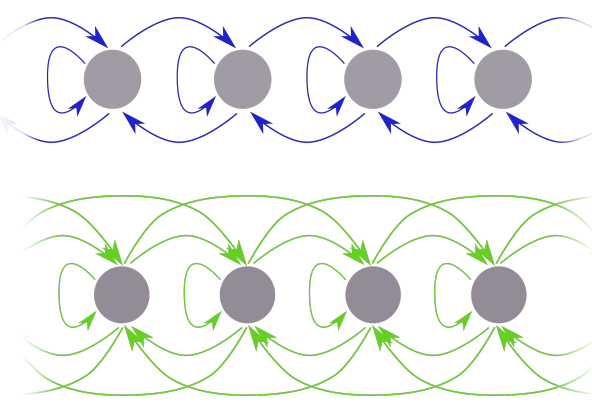

Figure 2: Connection graphs for networks $\mathcal{P}$ (blue) and $\mathcal{Q}$ (green)

Thus, if $i \neq j$, we can construct the gramian bispectrum element-wise from 111 via

$$
\operatorname{Bis}_{k}(i, j) \equiv \mathbf{d}_{\mathbb{J}}\left(\mathbf{u}_{i}, \mathbf{u}_{j}\right)=\frac{\sqrt{\left(\lambda_{\max }-\lambda_{i}\right)\left(\lambda_{\max }-\lambda_{j}\right)}}{\lambda_{\max }}
$$

while if $i=j$, we have

$$
\begin{aligned}
\operatorname{Bis}_{k}(i, j) \equiv \mathbf{d}_{\mathbb{J}}\left(\mathbf{u}_{i}, \mathbf{u}_{j}\right) & =\frac{1}{\lambda_{\max }}\left(\lambda_{j}+\sqrt{\left(\lambda_{\max }-\lambda_{i}\right)\left(\lambda_{\max }-\lambda_{j}\right)}\right) \\
& =1
\end{aligned}
$$

which is expected because of the law of cosines and the fact that $\mathbf{d}_{\mathbb{J}}\left(\mathbf{u}_{i}, \mathbf{u}_{j}\right)$ is the average integrated angular difference between inputs $\mathbf{u}_{i}$ and $\mathbf{u}_{j}(6)$.

Thus, it is quite straightforward to calculate the matrix $\mathbf{B i s}{ }_{k}$ for system $S_{k}$. As a second-order spectral characterization, we term $\mathbf{B i s}_{k}$ the gramian bispectrum of the system $S_{k}$.

\subsubsection{Example: Two Toeplitz Networks}

We consider a simple construction of two 20-dimensional linear time-invariant Toeplitz (i.e. the entries in any diagonal of the adjacency matrix are equal) networks $(\mathcal{P}$ and $\mathcal{Q})$, wherein each node of $\mathcal{P}$ has a self-connection and a bidirectional connection with its immediate neighbors, while $\mathcal{Q}$ has those connections as well as bi-directional connections with its secondary neighbors. The pattern of connectivity is schematized in Figure 2 The eigenspectra for the two networks are shown in Figure 3, and the gramian bispectra given in Figure 4 


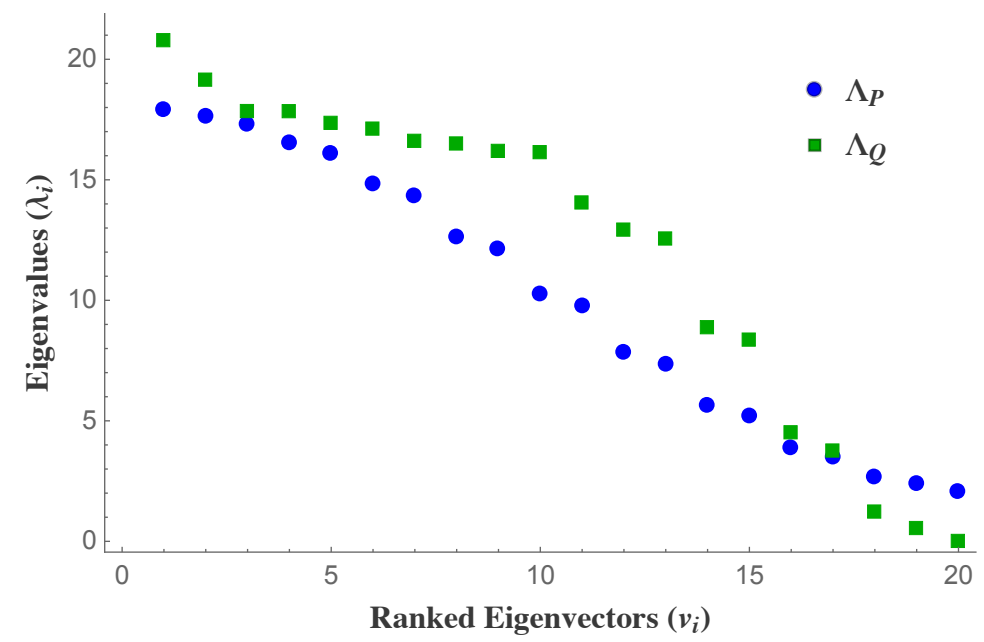

Figure 3: Spectra of $\mathbf{W}_{\mathcal{P}}^{-1}$ and $\mathbf{W}_{\mathcal{Q}}^{-1}$. The horizontal axis indexes the eigenvectors, which are sorted according to the magnitude of their associated real (since $\mathbf{W}^{-1}$ is real and symmetric) eigenvalues.
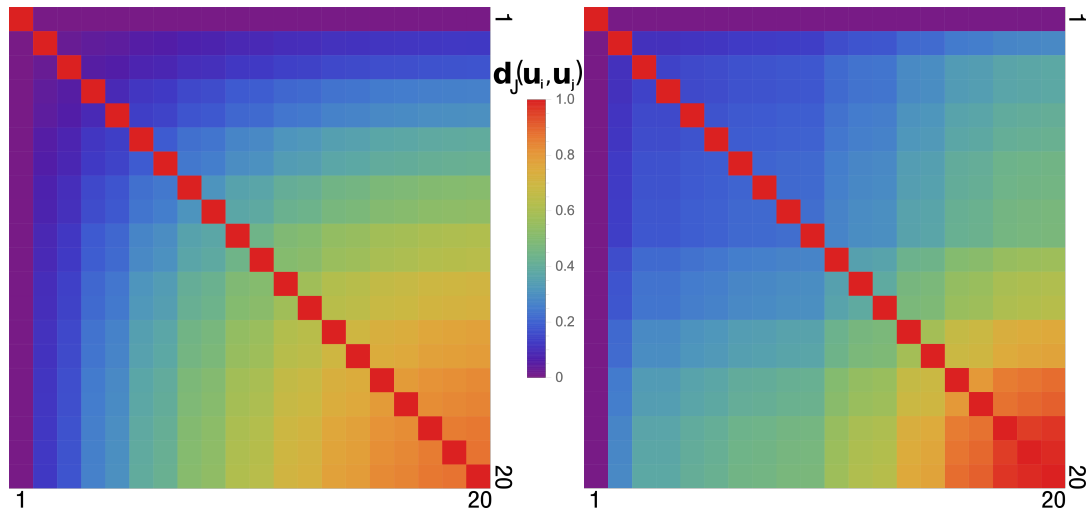

Figure 4: Array plots of gramian bispectra for networks $\mathcal{P}$ and $\mathcal{Q}$. As explained in detail in Section $3.1 . \mathbf{d}_{\mathbb{J}}\left(\mathbf{u}_{i}, \mathbf{u}_{j}\right)$ quantifies the average cosine of the angle between inputs $\mathbf{u}_{i}$ and $\mathbf{u}_{j}$, guiding the system to endpoints on the unit hypersphere in the directions of eigenvectors (of the gramian inverse $\left.\mathbf{W}^{-1}\right) \mathbf{v}_{i}$ and $\mathbf{v}_{j}$, respectively. Thus if $\mathbf{d}_{\mathbb{J}}\left(\mathbf{u}_{i}, \mathbf{u}_{j}\right)=1$, the inputs may be identical, which explains why the diagonal entries (where, obviously, $\mathbf{v}_{i}=\mathbf{v}_{j}$ ) are all equal to 1. 
As we examine Figure 4 , we notice that the overall contour of the ranked eigenvalues (Figure 3) is reflected in the bispectra, but that, especially for network $\mathcal{Q}$, there are subtleties reflected in the pairwise representation (i.e. the gramian bispectra) that can not be inferred from the eigenspectra, as we will explore below in Section III.C.

Since the gramian bispectrum is essentially a tabulation of within-system control flexibility which characterizes how a system may trade energy for a diversity of inputs to reach orthogonal points in state-space, worthy of special attention is the fact that the first row and column of the gramian bispectrum is always 0 (aside from the $\{1,1\}^{\text {th }}$ entry, whose value will be explained below), which, while apparent from (14), at first seems conceptually counterintuitive. The pairs of transfers whose minimum input orientation differences are encoded in the first row or column of the bispectrum are such that one of the state transfers employs a minimum energy input; that is $\lambda_{i}=\lambda_{\max }$ (first row) or $\lambda_{j}=\lambda_{\max }$ (first column). It would seem that, since the other input has an increasing amount of excess energy as we move along the first row or down the first column, that the orientation cost should decrease for the pair of inputs. That is, since $\lambda_{i_{1}} \geq \lambda_{i_{2}}$ when $i_{1}<i_{2}$, more excess energy is available for state transfers the farther we go along row 1 (or column 1 , substituting $j$ for $i$ ), which intuitively would seem to indicate greater input flexibility. However, this is not the case: when $\lambda_{i}=\lambda_{\max }$ or $\lambda_{j}=\lambda_{\max }$, it does not matter how much excess energy is provided for the other transfer, the best we can do is to be, on average, orthogonal. We formalize this notion in the following lemma:

Lemma 2. For inputs $\mathbf{u}_{1}(t)$ and $\mathbf{u}_{2}(t)$, guiding the system (over time horizon $\left.{ }_{165} t \in[0, T]\right)$ from the origin to $\mathbf{v}_{1}$ and $\mathbf{v}_{2}$, respectively, where $\mathbf{v}_{1}$ and $\mathbf{v}_{2}$ are eigenvectors of $\mathbf{W}^{-1}(T)$, and where exactly minimum energy is provided for at least one of the transfers, $\mathbf{d}_{\mathbb{J}}\left(\mathbf{u}_{1}, \mathbf{u}_{2}\right)=0$.

Proof 2. Let us examine (6) and assume we provide minimum energy for $\mathbf{u}_{1}$. Since $\mathbf{x}(0)$ is the origin and $\mathbf{x}(T)=\mathbf{v}_{1}$, where $\mathbf{v}_{1}$ is an eigenvector of $\mathbf{W}^{-1}(T)$, classical control theory (see [11], for example) tells us that minimum energy 
input $\mathbf{u}_{1}$ is given by: $\mathbf{u}_{1}(t)=\mathbf{B}^{\prime}(t) \mathrm{e}^{\mathbf{A}^{\prime}(T-t)} \mathbf{W}^{-1}(T) \mathbf{v}_{1}$. Substituting into (6), we obtain

$$
\begin{aligned}
& \mathbf{d}_{\mathbb{J}}\left(\mathbf{u}_{1}, \mathbf{u}_{2}\right)=\frac{1}{T \sqrt{\gamma_{\mathbf{u}_{1}} \gamma_{\mathbf{u}_{2}}}} \int_{0}^{T}\left(\mathbf{B}^{\prime}(\tau) \mathrm{e}^{\mathbf{A}^{\prime}(T-\tau)} \mathbf{W}^{-1}(T) \mathbf{v}_{1}\right)^{\prime} \mathbf{u}_{2}(\tau) \mathrm{d} \tau \\
& =\frac{1}{T \sqrt{\gamma_{\mathbf{u}_{1}} \gamma_{\mathbf{u}_{2}}}} \int_{0}^{T} \mathbf{v}_{1}^{\prime} \mathbf{W}^{-1}(T) \mathrm{e}^{\mathbf{A}(T-\tau)} \mathbf{B}(\tau) \mathbf{u}_{2}(\tau) \mathrm{d} \tau \\
& =\frac{1}{T \sqrt{\gamma_{\mathbf{u}_{1} \gamma_{\mathbf{u}_{2}}}}} \mathbf{v}_{1}^{\prime} \mathbf{W}^{-1}(T) \int_{0}^{T} \mathrm{e}^{\mathbf{A}(T-\tau)} \mathbf{B}(\tau) \mathbf{u}_{2}(\tau) \mathrm{d} \tau \\
& =\frac{1}{T \sqrt{\gamma_{\mathbf{u}_{1} \gamma_{\mathbf{u}_{2}}}}} \mathbf{v}_{1}^{\prime} \mathbf{W}^{-1}(T) \mathbf{v}_{2} \\
& =\frac{1}{T \sqrt{\gamma_{\mathbf{u}_{1} \gamma_{\mathbf{u}_{2}}}}} \lambda_{2} \mathbf{v}_{1}^{\prime} \mathbf{v}_{2}=0
\end{aligned}
$$

This somewhat surprising result means that any $\mathbf{u}_{2}$ which we choose to guide the system to $\mathbf{v}_{2}$ will have the same average angular difference $\left(\frac{\pi}{2}\right)$ from the minimum energy $\mathbf{u}_{1}$. Thus we obtain a sort of paradoxical freedom wherein we can do anything we want with $\mathbf{u}_{2}$ (subject to endpoint constraints) but we cannot improve on its similarity to $\mathbf{u}_{1}$.

\subsection{Geometric Interpretation}

Since the lengths of the axes of $\Xi$ (see (5), where for the present purposes we let $\left.c^{*}=1\right)$ are given by $\frac{1}{\sqrt{\lambda_{n}}}, \ldots, \frac{1}{\sqrt{\lambda_{1}}}$, we define $E_{1, i}$ to be the 2-D ellipse which results from a projection of $\Xi$ onto the axes given by $\mathbf{v}_{1}$ and $\mathbf{v}_{j}$, the eigenvectors associated with $\lambda_{1}$ and $\lambda_{j}$ (recall that $\lambda_{1}$ is the largest eigenvalues of $\mathbf{W}^{-1}(T)$, so we'll represent it, as above, by $\lambda_{\max }$ ). Then the eccentricity (we will call it $\left.\operatorname{ecc}_{E_{1, i}}\right)$ of $E_{1, i}$ is given by

$$
\begin{aligned}
\operatorname{ecc}_{E_{1, i}} & =\sqrt{1-\frac{\left(\frac{1}{\sqrt{\lambda_{\max }}}\right)^{2}}{\left(\frac{1}{\sqrt{\lambda_{i}}}\right)^{2}}}=\sqrt{1-\frac{\lambda_{i}}{\lambda_{\max }}} \\
& =\sqrt{\frac{\lambda_{\max }-\lambda_{i}}{\lambda_{\max }}}
\end{aligned}
$$


Choosing another such projected ellipse $E_{1, j}$ and its associated eccentricity $\operatorname{ecc}_{E_{1, j}}=\sqrt{\frac{\lambda_{\max }-\lambda_{j}}{\lambda_{\max }}}$, we notice that

$$
\begin{aligned}
e c c_{E_{1, i}} * e c c_{E_{1, j}} & =\sqrt{\frac{\lambda_{\max }-\lambda_{i}}{\lambda_{\max }}} \sqrt{\frac{\lambda_{\max }-\lambda_{j}}{\lambda_{\max }}} \\
& =\frac{\sqrt{\left(\lambda_{\max }-\lambda_{i}\right)\left(\lambda_{\max }-\lambda_{j}\right)}}{\lambda_{\max }} \\
& =\mathbf{d}_{\mathfrak{J}}\left(\mathbf{u}_{i}, \mathbf{u}_{j}\right) \quad(\text { Compare with }
\end{aligned}
$$

That is, the minimum relative orientation between inputs $\mathbf{u}_{i}$ and $\mathbf{u}_{j}$, utilizing total energy $\lambda_{\max }$ and driving the system to points on the unit hypersphere along the directions of eigenvectors $\mathbf{v}_{i}$ and $\mathbf{v}_{j}$, respectively, is the same as the multiple of the eccentricities of the 2-D ellipses generated from the projections of $\Xi$ onto $\mathbf{v}_{1}$ together with $\mathbf{v}_{i}$ and $\mathbf{v}_{j}$, respectively. Note that for different values of $c^{*}$ (again see (5) ) $\Xi$ is simply scaled and the eccentricity is not affected. Further, 180 a uniform scaling of the energy used by $\mathbf{u}_{i}$ and $\mathbf{u}_{j}$ does not affect $\mathbf{d}_{\mathbb{J}}\left(\mathbf{u}_{i}, \mathbf{u}_{j}\right)$ (see $20 p$ ). Thus 18 holds for $c^{*} \neq 1$. The geometric interpretation further highlights the fact that the gramian bispectrum furnishes a nice overall picture of system controllability in terms of energy, since these various eccentricities are exactly correlated with relative minimum energy requirements.

We now move on to concentrate on comparisons of gramian bispectra acrosssystem for different networks.

\subsection{Comparing Orientation Bispectra}

The various energy-based control metrics are useful primarily because of their comparative power; that is, given some energy metric and set of networks, we can easily rank the networks in terms of the energy costs. Since orientation difference is a more relativistic notion, we compare networks by calculating the gramian bispectral difference matrix

$$
\mathbf{D}_{\mathcal{P}, \mathcal{Q}}=\operatorname{Bis}_{\mathcal{P}}-\operatorname{Bis}_{\mathcal{Q}}
$$

for networks $\mathcal{P}$ and $\mathcal{Q}$. The $\{i, j\}^{\text {th }}$ entry of $\mathbf{D}_{\mathcal{P}, \mathcal{Q}}$ tells us the difference in relative orientation required (between the two networks) to accomplish the pair 

networks).

\subsubsection{Example: Toeplitz networks reveal informative bispectral differences}

We continue our analysis using the same Toeplitz networks $(\mathcal{P}$ and $\mathcal{Q})$ used in Example 1. If we examine Figure 3 we can make some conclusions about the relative controllability of networks $\mathcal{P}$ and $\mathcal{Q}$, namely that $\mathcal{P}$ requires less energy to steer in most directions of the state space. For only the three 'easiest' directions is $\mathcal{Q}$ more energy-efficient. This may be all we desire to know in comparing the two systems if energy is our primary consideration. However, if we know that we have energy to spare, we can examine the bispectral difference of the two networks to evaluate their relative efficiency in trading excess energy for input flexibility.

To make a fair comparison of the two networks, we will rescale the eigenspectra so that their maximum eigenvectors are equal-that is, $\lambda_{\mathcal{P}, 1}=\lambda_{\mathcal{Q}, 1}^{*}$, where $\lambda_{\mathcal{Q}, 1}^{*} \in \Lambda_{\mathcal{Q}}^{*}$, the transformed eigenspectrum for network $\mathcal{Q}$. Note that this will not affect the gramian bispectra, for, if we scale the spectrum (i.e. all of the eigenvalues) of $\mathbf{W}^{-1}$ by some constant $\alpha$ and evaluate 14 , we have

$$
\begin{aligned}
\mathbf{d}_{\mathbb{J}}^{*}\left(\mathbf{u}_{i}, \mathbf{u}_{j}\right) & =\frac{\sqrt{\left(\alpha \lambda_{\max }-\alpha \lambda_{i}\right)\left(\alpha \lambda_{\max }-\alpha \lambda_{j}\right)}}{\alpha \lambda_{\max }} \\
& =\mathbf{d}_{\mathbb{J}}\left(\mathbf{u}_{i}, \mathbf{u}_{j}\right)
\end{aligned}
$$

Now, let $\lambda_{\mathcal{P}, \max }$ and $\lambda_{\mathcal{Q}, \max }$ be the maximal inverse gramian $\left(\mathbf{W}^{-1}\right)$ eigenvalues of networks $\mathcal{P}$ and $\mathcal{Q}$, respectively. As in (4), let us denote the ordered spectra of $\mathbf{W}^{-1}$ for the two networks by $\Lambda_{\mathcal{P}}$ and $\Lambda_{\mathcal{Q}}$, respectively. By 20 , we can let $\Lambda_{\mathcal{Q}}^{*}=c \Lambda_{\mathcal{Q}}$, where

$$
c=\frac{\lambda_{\mathcal{P}, \max }}{\lambda_{\mathcal{Q}, \max }},
$$

such that the rescaled spectrum $\Lambda_{\mathcal{Q}}^{*}$ results in no change to $\mathcal{Q}$ 's gramian bispec- 


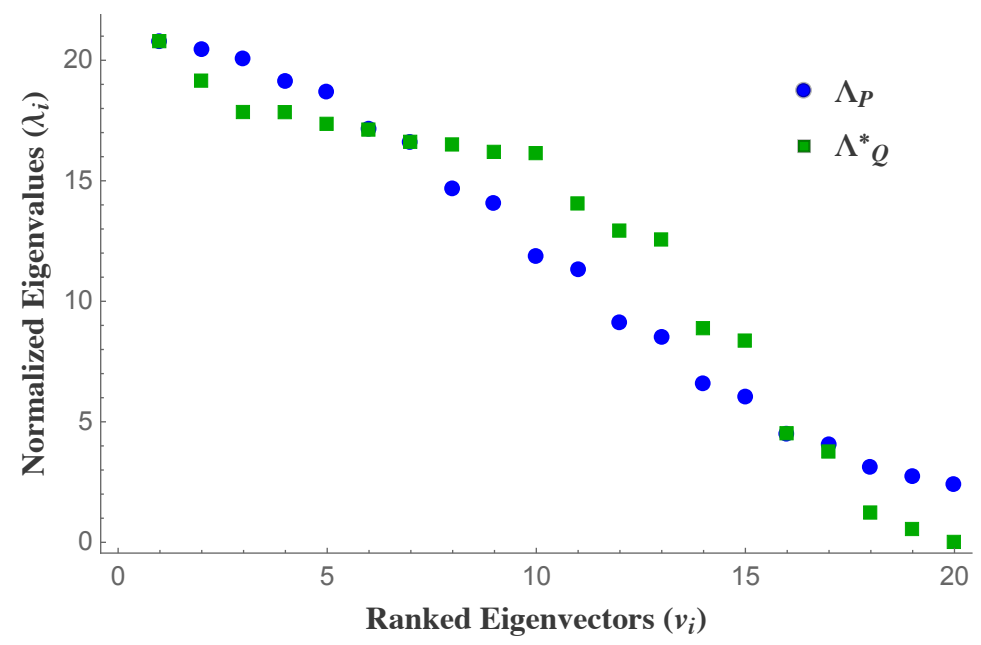

Figure 5: Spectra of $\mathcal{P}$ and $\mathcal{Q}$, with the latter normalized by $c$ (see 21) so that the largest eigenvalues agree. This normalization does not affect the bispectral analysis (see 222). As in Figure 3 the horizontal axis indexes the eigenvectors, which are sorted according to the magnitude of their associated eigenvalues.

trum (that is, $\mathbf{B i s}_{\mathcal{Q}}^{*}=\mathbf{B i s}_{\mathcal{Q}}$ ). Then we have, for network $\mathcal{Q}$,

$$
\begin{aligned}
\operatorname{Bis}_{\mathcal{Q}_{i, j}} & =\mathbf{d}_{\mathbb{J}}\left(\mathbf{u}_{i}, \mathbf{u}_{j}\right) \\
& =\frac{\sqrt{\left(\lambda_{\mathcal{Q}, \text { max }}-\lambda_{\mathcal{Q}, i}\right)\left(\lambda_{\mathcal{Q}, \max }-\lambda_{\mathcal{Q}, j}\right)}}{\lambda_{\mathcal{Q}, \max }} \\
& =\frac{\sqrt{\left(\lambda_{\mathcal{P}, \max }-c \lambda_{\mathcal{Q}, i}\right)\left(\lambda_{\mathcal{P}, \max }-c \lambda_{\mathcal{Q}, j}\right)}}{\lambda_{\mathcal{P}, \max }}
\end{aligned}
$$

This rescaling, applied to our networks $\mathcal{P}$ and $\mathcal{Q}$, results in spectra as shown in Figure 5 .

The reason this rescaling is advantageous is that it allows us to investigate how different spectral profiles are manifest in the respective gramian bispectra, and ultimately in the bispectrum difference matrix. For example, it is evident from (14) that there must exist crossings in the normalized spectra in order for there to be any nontrivial comparison. For, if, without loss of generality, $\lambda_{\mathcal{P}, i}>\lambda_{\mathcal{Q}, i}^{*} \forall i \in\{2, \ldots, n\}$ (recall that the normalization ensures that $\lambda_{\mathcal{P}, \max }=$ 


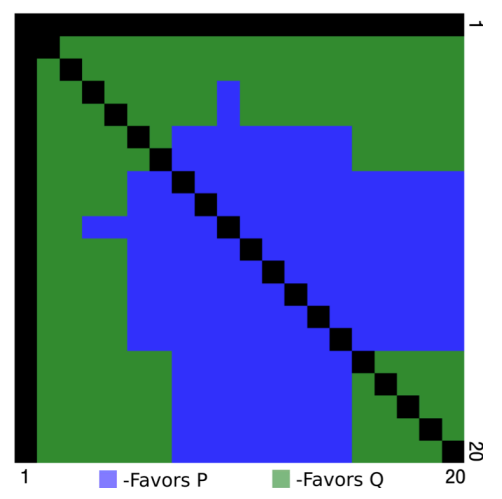

Figure 6: A visualization of bispectrum difference matrix $\mathbf{D}_{\mathcal{P}, \mathcal{Q}}$, a tool for comparing the excess energy utility of the two networks. The $\{i, j\}^{\text {th }}$ entry is colored blue where network $\mathcal{P}$ has the lower required angular input separation for maneuvers to $\mathbf{v}_{i}$ and $\mathbf{v}_{j}$, and colored green if network $\mathcal{Q}$ is better in this respect.

$\left.\lambda_{\mathcal{Q}, \max }^{*}=\lambda_{\max }\right)$ then

$$
\begin{aligned}
& \mathbf{D}_{\mathcal{P}, Q_{i, j}}=\mathbf{B i s}_{P_{i, j}}-\mathbf{B i s}_{Q_{i, j}} \\
&= \frac{\sqrt{\left(\lambda_{\mathcal{P}, \max }-\lambda_{\mathcal{P}, i}\right)\left(\lambda_{\mathcal{P}, \max }-\lambda_{\mathcal{P}, j}\right)}}{\lambda_{\mathcal{P}, \max }}- \\
& \frac{\sqrt{\left(\lambda_{\mathcal{Q}, \text { max }}^{*}-\lambda_{\mathcal{Q}, i}^{*}\right)\left(\lambda_{\mathcal{Q}, \max }^{*}-\lambda_{\mathcal{Q}, j}^{*}\right)}}{\lambda_{\mathcal{Q}, \max }^{*}} \frac{\sqrt{\left(\lambda_{\max }-\lambda_{\mathcal{P}, i}\right)\left(\lambda_{\max }-\lambda_{\mathcal{P}, j}\right)}-\sqrt{\left(\lambda_{\max }-\lambda_{\mathcal{P}, i}\right)\left(\lambda_{\max }-\lambda_{\mathcal{P}, j}\right)}}{\lambda_{\max }} \\
&<0 \quad \forall\{i, j\} i \in\{2, \ldots, n\}, j \in\{2, \ldots, n\} \text { where } i \neq j
\end{aligned}
$$

and thus the bispectrum difference matrix would reveal that network $\mathcal{Q}$ is easier to control (in terms of input flexibility) for all pairwise maneuvers. Clearly, $\mathcal{Q}$ would be easier to control in terms of minimum energies as well. But a more complex picture may emerge when the normalized spectra have crossings. The fact that 14 is a nonlinear function of the magnitudes of $\lambda_{\max }, \lambda_{i}$, and $\lambda_{j}$ makes it impossible to predict, upon inspection of the eigenspectra, whether $\mathbf{D}_{\mathcal{P}, Q_{i, j}}$ will be positive or negative when either $\left(\lambda_{\mathcal{P}, i}>\lambda_{\mathcal{Q}, i}^{*}\right) \wedge\left(\lambda_{\mathcal{P}, j}<\lambda_{\mathcal{Q}, j}^{*}\right)$ or $\left(\lambda_{\mathcal{P}, i}<\lambda_{\mathcal{Q}, i}^{*}\right) \wedge\left(\lambda_{\mathcal{P}, j}>\lambda_{\mathcal{Q}, j}^{*}\right)$ (where $\wedge$ is the logical 'and' operator).

We seek now to understand how this bispectral difference analysis can be 
interpreted to infer relative controllability strengths and weaknesses, using Figure 6 (which was constructed by using the sign function on the entries of $\mathbf{D}_{\mathcal{P}, \mathcal{Q}}$ ) as an example. The first thing that strikes us in examining Figure 6 is that for most pairs of maneuvers wherein one has relatively little excess energy (this corresponds to the first rows and columns of the matrix), network $\mathcal{Q}$ has lower pairwise orientation cost, as represented by the large swaths of green in the early rows and columns of Figure 6. Now, examining Figure 5, we see there is a crossing at the $6^{\text {th }}$ eigenvalue, so we would expect to see a change in the difference matrix at the sixth row/column (recall that $\mathrm{D}_{\mathcal{P}, \mathcal{Q}}$ is always symmetric). Indeed we do see green give way to blue, representing the fact that network $\mathcal{P}$ has a lower orientation cost for pairwise maneuvers to eigenvectors with associated eigenvalues in the 'middle' section of the spectrum.

However, the change is more complex than can be inferred from the spectra alone. Notice, in Figure 6, the protrusions of blue into the green section which appears in the $4^{\text {th }}$ and $5^{\text {th }}$ rows (columns) and the $10^{\text {th }}$ column (row). This indicates that, for pairwise maneuvers where one of the maneuvers involves the $10^{\text {th }}$ eigenvector $\left(\mathbf{v}_{10}\right)$ and the other involves $\mathbf{v}_{i} i \in\{4,5\}$, network $\mathcal{P}$ has the better excess energy utility, while if the $\mathbf{v}_{i} i \in\{4,5\}$ are paired with any other eigenvector, network $\mathcal{Q}$ is more favorable. Clearly, by examining only the sign of the entries of $\mathbf{D}_{\mathcal{P}, \mathcal{Q}}$, we limit the possible depth of the analysis. If we include magnitudes in our consideration, an even richer characterization may emerge. For example, it may be that network $\mathcal{P}$ is better (according to our objective) for only a few pairs of directions, but for those pairs it is much better. If our control objectives prioritize input flexibility in traversing these directions of the state space, we may deem $\mathcal{P}$ the more efficient network.

If, on the other hand, we desire an even further simplification, we could condense the information provided by the bispectral difference into one summary

240 statistic by simply summing over the entries of $\mathrm{D}_{\mathcal{P}, \mathcal{Q}}$ to obtain an overall picture of which network best utilizes its excess energy. However, much of the nuanced interpretation would be lost by doing so. 


\section{Conclusions and Future Work}

We have introduced a mathematical construct, the gramian bispectrum,

which provides a characterization of how a system trades off energy for control versatility in the sense of an angular distance metric. We remark that, while certainly the standard (energy) spectra give some indications as to what we might expect to see in the relative gramian bispectral matrices (i.e. the $\mathbf{D}_{\mathcal{P}, \mathcal{Q}}$ ), these matrices themselves furnish a richer picture of the relative control 250 versatility for maneuvers in the different (in these cases orthogonal) directions. We suggest that the interpretive possibilities of the gramian bispectrum concept hold potential in the study and comparison of linear systems and networks.

\section{Acknowledgement}

ShiNung Ching holds a Career Award at the Scientific Interface from the Burroughs-

Wellcome Fund. This work was partially supported by AFOSR 15RT0189, NSF ECCS 1509342 and NSF CMMI 1537015, from the US Air Force Office of Scientific Research and the US National Science Foundation, respectively.

[1] G. Yan, J. Ren, Y.-C. Lai, C.-H. Lai, B. Li, Controlling complex networks: how much energy is needed?, Physical review letters 108 (21) (2012) 218703.

[2] G. Hu, E. Davison, Real controllability/stabilizability radius of LTI systems 49 (2) (2004) 254-257. doi:10.1109/TAC.2003.822871.

[3] G. Hu, E. J. Davison, Real controllability/stabilizability radius of LTI systems, IEEE Transactions on Automatic Control 49 (2) (2004) 254-257.

[4] F. Pasqualetti, S. Zampieri, On the controllability of isotropic and anisotropic networks.

[5] F. Pasqualetti, S. Zampieri, F. Bullo, Controllability metrics, limitations and algorithms for complex networks, IEEE Transactions on Control of Network Systems 1 (1) (2014) 40-52. doi:10.1109/TCNS.2014.2310254. 
[6] B. Dumitrescu, B. Sicleru, R. Stefan, Computing the controllability radius: a semi-definite programming approach, IET Control Theory \& Applications 3 (6) (2009) 654-660. doi:10.1049/iet-cta.2008.0169 URL http://ieeexplore.iee.org/stamp/stamp.jsp?arnumber= 4976843

[7] F. L. Cortesi, T. H. Summers, J. Lygeros, Submodularity of energy related controllability metrics, ArXiv preprint arXiv:1403.6351.

[8] F. Pasqualetti, S. Zampieri, F. Bullo, Controllability metrics, limitations and algorithms for complex networks, IEEE Transactions on Control of Network Systems 1 (1) (2014) 40-52.

口 [9] G. Kumar, D. Menolascino, M. Kafashan, S. Ching, Controlling linear networks with minimally novel inputs, in: American Control Conference (ACC), 2015, 2015, pp. 5896-5900. doi:10.1109/ACC.2015.7172264.

1 U URL http://ieeexplore.iee.org/stamp/stamp.jsp?arnumber= 7172264

[10] D. Menolascino, G. Kumar, S. Ching, Endpoint-based discriminability of minimum energy inputs, in: 2016 American Control Conference, 2016.

[11] R. W. Brockett, Finite dimensional linear systems, Wiley New York, 1970. 